DRAFT VERSION NOVEMBER 8, 2018

Preprint typeset using $\mathrm{LT}_{\mathrm{E}} \mathrm{X}$ style emulateapj v. 11/26/04

\title{
CONSTRAINTS ON THE EMISSION AND VIEWING GEOMETRY OF THE TRANSIENT ANOMALOUS X-RAY PULSAR XTE J1810-197
}

\author{
Rosalba Perna ${ }^{1}$ And E. V. GotThelF ${ }^{2}$ \\ Draft version November 8, 2018
}

\begin{abstract}
The temporal decay of the flux components of Transient Anomalous X-ray Pulsar XTE J1810-197 following its 2002 outburst presents a unique opportunity to probe the emission geometry of a magnetar. Toward this goal, we model the magnitude of the pulsar's modulation in narrow spectral bands over time. Following previous work, we assume that the post-outburst flux is produced in two distinct thermal components arising from a hot spot and a warm concentric ring. We include general relativistic effects on the blackbody spectra due to gravitational redshift and light bending near the stellar surface, which strongly depend on radius. This affects the model fits for the temperature and size of the emission regions. For the hot spot, the observed temporal and energy-dependent pulse modulation is found to require an anisotropic, pencil-beamed radiation pattern. We are able to constrain an allowed range for the angles that the line-of-sight $(\psi)$ and the hot spot pole $(\xi)$ make with respect to the spin-axis. Within errors, this is defined by the locus of points in the $\xi-\psi$-plane that lie along the line $(\xi+\beta(R))(\psi+\beta(R)) \approx$ constant, where $\beta(R)$ is a function of the radius $R$ of the star. For a canonical value of $R=12 \mathrm{~km}$, the viewing parameters range from $\psi=\xi=37^{\circ}$ to $(\psi, \xi)=\left(85^{\circ}, 15^{\circ}\right)$. We discuss our results in the context of magnetar emission models.
\end{abstract}

Subject headings: pulsars: individual (XTE J1810-197) — stars: neutron — X-rays: stars

\section{INTRODUCTION}

Anomalous X-ray Pulsars (AXPs) are peculiar high-energy pulsars whose observed luminosity greatly exceeds that which can be supplied by their rotational energy losses. These pulsars occupy a narrow range of spin periods $(P \sim 2-12$ sec) and are spinning down rapidly compared to the rotationpowered pulsars. For the vacuum dipole model, the timing properties of these pulsars imply an enormous magnetic field, $B \sim 10^{14}-10^{15} \mathrm{G}$. These relatively rare objects $(\sim 10 \mathrm{com}-$ pared to $\sim 1700$ catalogued radio pulsars), generally display sinusoidal modulation in their pulsed flux, with a wide range of amplitudes $(\sim 10-80 \%)$ and are likely young $\left(<10^{4} \mathrm{yrs}\right)$, as more than half are associated with supernova remnants (see Kaspi 2007 for a recent review). AXPs can be understood within the context of the magnetar model developed by Duncan \& Thompson (1992) to explain the burst phenomenology of Soft $\gamma$-ray Repeaters (SGRs). The excess emission from both AXPs and SGRs, collectively referred to as magnetars, is powered by the decay of their extreme magnetic fields. This is suggested by the relatively high temperatures of their thermal emission $(k T \approx 0.4-0.7 \mathrm{keV}$ for blackbody fits), and frequent rapid $(<0.1 \mathrm{~s})$ burst activity. The geometry and the properties of the observed emission from the magnetars is of great interest for understanding how this activity arises.

The recent discovery of an AXP fading from a long duration outburst offers the unique opportunity to probe the magnetar emission geometry evolution during this event. The $P=5.54 \mathrm{~s}$ Transient AXP (TAXP) XTE J1810-197 was discovered in January 2003 by Ibrahim et al. (2004) using the Rossi $X$ ray timing explorer ( $R X T E$ ) following a large eruption. Subsequently its flux decayed exponentially $(\tau \approx 900 \mathrm{~d})$ nearly back to a quiescent flux level as determined from serendipitously archival X-ray observations (Gotthelf et al. 2004).

\footnotetext{
${ }^{1}$ JILA and Department of Astrophysical and Planetary Sciences, University of Colorado, Boulder, CO, 80309

${ }^{2}$ Columbia Astrophysics Laboratory, Columbia University, New York, NY 10027
}

The earlier measurements indicate that the TAXPs $2-10 \mathrm{keV}$ flux had increased by two orders of magnitude. However, of great interest, the quiescent luminosity is 100 times lower than for the well-established AXPs and SGRs, suggesting a large unidentified population of neutron stars (Gotthelf et al. 2004). In contrast, the magnetic field strength of XTE J1810-197, $B=3 \times 10^{14} \mathrm{G}$ as inferred from its spin-down properties, is typical of the magnetars.

The flux and pulse evolution of XTE J1810-197 were monitored with the XMM-Newton X-ray observatory at roughly biyearly intervals starting Sept. 2003, yielding a total of seven epochs through Mar. 2006. The complete set of observations, together with their spectral modeling and interpretation, is described in detail by Gotthelf \& Halpern (2007), with the earlier observations reported in Gotthelf \& Halpern (2005) and Halpern \& Gotthelf (2005). While analysis of phaseaveraged spectra alone cannot distinguish among competing models for the AXP emission type and geometry, the addition of the steady change of the spectrum and pulse modulation over time greatly increases the diagnostic power.

In this paper we present a detailed model for the energy dependent pulse phase from XTE J1810-197. This model accounts for the viewing geometry and surface emission distribution. We include the general relativistic effects of light deflection and gravitational redshift and allow for anisotropic emission. We apply this model to a set of X-ray data acquired during the temporal evolution of the flux from XTE J1810-197. This allows us to constrain the underlying emission geometry and radiation properties of this transient magnetar.

\section{TIME-DEPENDENT FLUX MODELING OF XTE J1810-197}

\subsection{Model Motivations}

Since their discovery, spectra of magnetars have been fitted with a variety of models, generally including two components, such as blackbody plus power-law, atmosphere plus power-law (Perna et al. 2001a; Skinner et al. 2006), or thermal plus resonant cyclotron scattering (Rea et al. 2007), or 
more recently, a magnetized atmosphere model with the inclusion of scattering (Guver et al. 2007). From the point of view of phase-averaged spectral analysis alone, these models are generally statistically acceptable, and therefore none can be ruled out a priori.

Phase-resolved modeling of the observed modulation, however, can provide a much stronger constraint. This is particularly true for the case of XTE J1810-197, given the wealth of data available at different epochs, while the object is cooling. From the point of view of being able to reproduce the observed time-dependent energy behavior of the pulsed fractions, the double blackbody model (made up of a hot and a warm component) put forward by Gotthelf \& Halpern (2005) is quite promising, at least from a qualitative point of view. In fact, if, in the same energy band, the warm component is less pulsed than the hot one, then, as the hot component drops in flux faster than the warm one (as found in their fitting), the pulse fraction will tend to decline, as observed. Also, in their modeling, the area of the warm component, which becomes more luminous than the hot component after the fourth epoch, increases at later times. ${ }^{3}$ This, again, tends to produce a decline in the pulse modulation and counteract the increase that would otherwise have, due to the decreasing temperature.

While it is tempting to model the thermal components with detailed magnetized atmospheres (e.g. van Adelsberg \& Lai 2006), these models might be problematic for the case of XTE J1810-197 following its outburst. Invoking dissipation of a twist in the magnetic field lines (Beloborodov \& Thompson 2007), the field in the emitting region is likely to have significant non-normal components. While the "twist model" nicely predicts the timescale of the outburst decay, the nonnormal surface magnetic fields (i.e. magnetic fields that emerge from the star surface at oblique angles) have yet to be fully realized in the magnetized atmosphere models. Since the predicted amplitude of the flux modulation strongly depends on the local magnetic field direction, by assuming a magnetized atmosphere model for the thermal components we would introduce an a priori bias in our results.

In this work, we prefer to take a more empirical approach by starting with the distribution of the emitted radiation over the stellar surface and allowing a degree of anisotropy (beaming factor) in the thermal (blackbody) components (following the methods of Pechenick et al. 1993; DeDeo et al. 2000; Perna et al. 2001b), and leaving the beaming factor as one of the model parameters. This approach turns out to be very valuable in that we can use the energy-dependency of the pulsed fractions, together with their variation with time as the emitting region cools, to constrain both the viewing geometry and the beaming properties of the radiation simultaneously $(\$ 3)$. Our results can therefore be used as a guide for further theoretical modeling aimed at understanding the detailed mechanisms that produce the observed thermal radiation.

\subsection{The Emission Model}

Our method for parameterizing the surface emission from XTE J1810-197 follows the example developed by Pechenick et al. (1993) with some generalizations. For our numerical

\footnotetext{
3 This area increase might either be real, or a result of the fact that the warm component cannot be straightforwardly separated in the fits from the underlying surface emission, to which it approaches at later times. Either way, the pulse modulation in this model is expected to decrease with time, since the surface emission of the neutron star, if its temperature distribution traces that of a dipolar magnetic field, is not expected to be highly modulated (DeDeo et al. 2001).
}

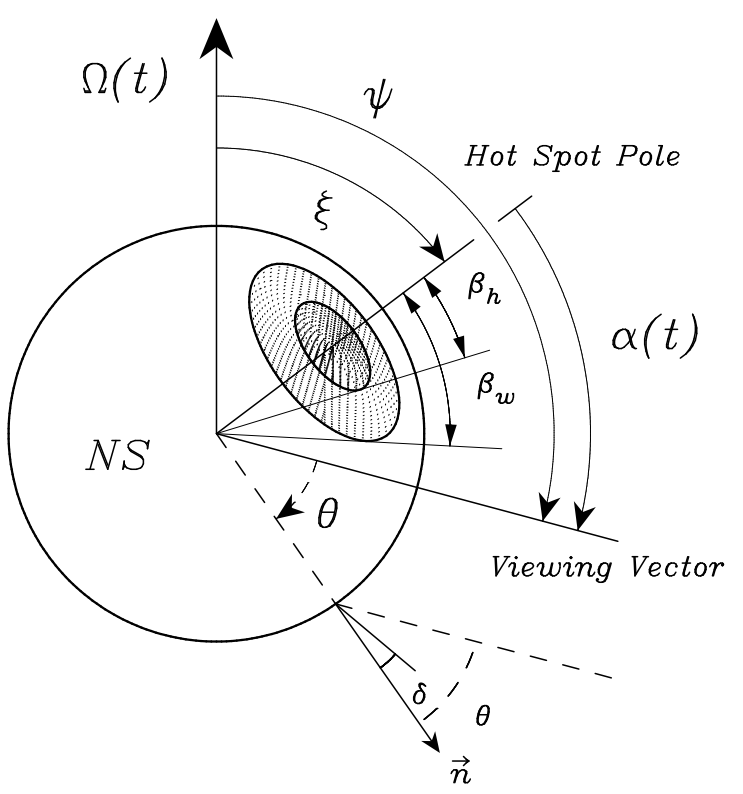

FIG. 1.- Emission geometry on the surface of the neutron star (NS) for the model presented herein. A hot spot of temperature $T_{h}$ and angular size $\beta_{h}$ is surrounded by a warm ring of temperature $T_{w}$ with outer angular size $\beta_{w}$. As the neutron star (NS) rotates with angular velocity $\Omega(t)$, the angle $\alpha(t)$ is a function of the phase angle $\gamma(t)=\Omega(t) t$, and the angles $\psi$ and $\xi$ between spin axis and viewing vector, and between spin axis and emission pole, respectively. Due to general relativistic effects, a photon emitted at a colatitude $\theta$ on the star's surface which reaches the observer must be emitted at an angle $\delta$ with respect to the star's normal at that point.

modeling we consider emission from a hot spot of temperature $T_{h}$ and angular radius $\beta_{h}$ surrounded by a warm ring of temperature $T_{w}$ and outer radius $\beta_{w}$

We indicate with $\alpha$ the angle that the axis of the hot spot makes with respect to the line of sight. This depends on the phase angle $\gamma(t)=\Omega(t) t$, as the star rotates with angular velocity $\Omega(t)$. If $\xi$ is the angle between the spot axis and the rotation axis, and $\psi$ the angle between the observer's direction and the rotation axis, then the angle $\alpha$ is given by

$$
\alpha(t)=\arccos (\cos \psi \cos \xi+\sin \psi \sin \xi \cos \gamma(t)) .
$$

The geometry is illustrated in Figure 1. The surface of the star is described by the angular spherical coordinates $(\theta, \phi)$, and the coordinate system is chosen so that the $z$ axis is in the direction of the line of sight to the observer. This is a natural choice of coordinate system for our problem, since the observed flux is produced by all the photons that reach the observer at $\infty$ along that axis (e.g. Pechenick et al. 1993; Page 1995). Note that, if there were no general relativistic effects, a photon emitted at colatitude $\theta$ on the star would only reach the observer if it were emitted at an angle $\delta=\theta$ with the normal to the surface of the star. Because of general relativistic effects, however, a photon emitted at a colatitude $\theta$ will get to the observer only if emitted at an angle $\delta$ with respect to the surface normal (see Fig.1), where the relation between the two angles is given by the ray-tracing function ${ }^{4}$ (Pechenick et al. 1993; Page 1995)

$$
\theta(\delta)=\int_{0}^{R_{s} / 2 R} x d u / \sqrt{\left(1-\frac{R_{s}}{R}\right)\left(\frac{R_{s}}{2 R}\right)^{2}-(1-2 u) u^{2} x^{2}}
$$

\footnotetext{
${ }^{4}$ To improve the computational efficiency of the above equation we use the approximation presented in Beloborodov (2002).
} 
having defined $x \equiv \sin \delta$. Here, $R / R_{s}$ is the ratio of the stellar to Schwarzschild radius, $R_{s}=2 G M / c^{2}$ (we assume $\left.M=1.4 M_{\odot}\right)$.

The hot spot is described by the conditions:

$$
\theta \leq \beta_{h}, \quad \text { if } \alpha=0
$$

and

$$
\left\{\begin{array}{l}
\alpha-\beta_{h} \leq \theta \leq \alpha+\beta_{h} \\
2 \pi-\phi_{p}^{h} \leq \phi \leq \phi_{p}^{h}
\end{array} \text { if } \alpha \neq 0 \text { and } \beta_{h} \leq \alpha\right.
$$

where

$$
\phi_{p}^{h}=\arccos \left[\frac{\cos \beta_{h}-\cos \alpha \cos \theta}{\sin \alpha \sin \theta}\right] .
$$

On the other hand, it is identified through the condition

$$
\theta \leq \theta_{*}^{h}\left(\alpha, \beta_{h}, \phi\right), \quad \text { if } \alpha \neq 0 \text { and } \beta_{h}>\alpha,
$$

where the outer boundary $\theta_{*}^{h}\left(\alpha, \beta_{h}, \phi\right)$ of the spot is computed by numerical solution of the equation

$$
\cos \beta_{h}=\sin \theta_{*}^{h} \sin \alpha \cos \phi+\cos \theta_{*}^{h} \cos \alpha .
$$

Similarly, the warm ring is described on the star surface through the conditions

$$
\beta_{h}<\theta \leq \beta_{w}, \quad \text { if } \alpha=0
$$

and

$$
\left\{\begin{array}{l}
\alpha-\beta_{w} \leq \theta \leq \alpha+\beta_{w} \text { and } \alpha+\beta_{h} \leq \theta \leq \alpha-\beta_{h} \\
2 \pi-\phi_{p}^{w} \leq \phi \leq \phi_{p}^{w} \text { and } \phi_{p}^{h} \leq \phi \leq 2 \pi-\phi_{p}^{h}
\end{array}\right.
$$

if $\alpha \neq 0$ and $\beta_{w} \leq \alpha$. In the above equation, $\phi_{p}^{w}$ has the same functional form as $\phi_{p}^{h}$ in Eq (5), except for the substitution $\beta_{h} \rightarrow \beta_{w}$. Finally, if $\alpha \neq 0$ and $\beta_{w}>\alpha$, the ring is identified by the condition

$$
\theta_{*}^{h}\left(\alpha, \beta_{h}, \phi\right)<\theta \leq \theta_{*}^{w}\left(\alpha, \beta_{w}, \phi\right),
$$

where, again, the outer boundary of the ring $\theta_{*}^{w}\left(\alpha, \beta_{w}, \phi\right)$ is found by numerical solution of Eq. (7), but with the replacement $\beta_{h} \rightarrow \beta_{w}$.

In the following we assume a blackbody emission model for both the hot spot and warm ring, characterized by a uniform temperature ( $T_{h}$ or $T_{w}$, respectively) over their stellar surface. As discussed above, we allow for the radiation from the two regions to be anisotropic, and parameterize the beaming of their local emission through the functions $f_{h}(\delta) \propto \cos ^{n_{h}}(\delta)$ (hot spot) and $f_{w}(\delta) \propto \cos ^{n_{w}}(\delta)$ (warm ring). This choice was initially motivated by the consideration that the hot spots are likely associated with regions of larger conductivity, where the magnetic field lines would be close to perpendicular to the surface of the star. This would produce an enhanced emissivity at small $\delta$. Our analysis (§3.2) then confirmed the validity of this choice by demonstrating that the modulation of the hottest region does indeed require a pencil-type anisotropic beaming pattern.

The observed spectrum as a function of phase angle $\gamma$ is then obtained by integrating the local emission over the observable surface of the star, accounting for the gravitational redshift of the radiation (Page 1995)

$$
\begin{gathered}
F\left(E_{\infty}, \gamma\right)=\frac{2 \pi}{c h^{3}} \frac{R_{\infty}^{2}}{D^{2}} E_{\infty}^{2} e^{-N_{\mathrm{H}} \sigma\left(E_{\infty}\right)} \int_{0}^{1} 2 x d x \\
\times \int_{0}^{2 \pi} \frac{d \phi}{2 \pi} I_{0}(\theta, \phi) n\left[E_{\infty} e^{-\Lambda_{s}} ; T(\theta, \phi)\right],
\end{gathered}
$$

in units of photons $\mathrm{cm}^{-2} \mathrm{~s}^{-1} \mathrm{keV}^{-1}$. In the above equation, the radius and energy as observed at infinity are given by $R_{\infty}=$ $R e^{-\Lambda_{s}}$, and $E_{\infty}=E e^{\Lambda_{s}}$, where $R$ is the star radius, $E$ is the energy emitted at the star surface, and we have defined $\Lambda_{s}$ as,

$$
e^{\Lambda_{s}} \equiv \sqrt{1-\frac{R_{s}}{R}}
$$

For the spectral function, given by $n(E, T)=$ $1 /[\exp (E / k T)-1]$, the temperature $T(\theta, \phi)$ is equal to $T_{h}$ if $\{\theta, \phi\}$ satisfy any of the conditions (3) through (7), and it is given by $T(\theta, \phi)=T_{w}$ if any of the conditions (8) through (10) holds true. Correspondingly, the weighted intensity $I_{0}(\theta, \phi)$ is given by the functions $f_{h}$ or $f_{w}$ depending on whether the variables $\{\theta, \phi\}$ are inside the hot or warm region, respectively.

The phase-averaged flux is readily computed as $F_{\text {ave }}\left(E_{\infty}\right)=$ $1 / 2 \pi \int_{0}^{2 \pi} d \gamma F\left(E_{\infty}, \gamma\right)$. The phase dependence $\gamma$ in Eq. (11) comes from the viewing angles implicit in $\alpha(t)$ and from the series of conditions (3) through (10). Note that, as the star rotates, the only angle on which the flux depends is $\alpha(t)$, the angle that the line of sight makes with the axis of the spots. Since, in the magnetar model, the spots are likely to be correlated to regions with an enhanced magnetic activity, the angle $\alpha(t)$ can also be considered as the (phase-dependent) angle between the line of sight and a magnetically active region on the star during and following the outburst. When the star returns to quiescence, the temperature distribution on the star will reflect the overall magnetic field configuration. For most AXPs, the quiescent emission cannot be produced by a temperature distribution following a dipolar magnetic field (De Deo, Psaltis \& Narayan 2000); in the case of XTE J1810197 , a detailed study of the quiescent emission, once the contamination from the heated regions has completely subsided, will be able to determine the detailed structure of the surface temperature distribution, and hence reconstruct the magnetic field structure in quiescence. This study will be performed in a forthcoming paper. However, a preliminary investigation of the softest energy band in the latest data set (where the surface emission from the rest of the star is likely to be dominant) shows that the maximum of the pulsed emission remains in phase with the maximum in the hardest energy band (still dominated by the heated region). This result shows that the maximum of the quiescent emission comes from the region where the outburst occurred. Therefore, the active region is likely to be associated with an enhanced magnetic field strength in quiescence. If the underlying $B$ field is dipolar (or close to such), then the spot axis in our paper also represents the dipole magnetic field axis, and therefore the angle $\alpha(t)$ with respect to the $z$-axis (observer's viewing direction) would naturally be associated with the angle that the magnetic axis forms with respect to our line of sight as the star rotates.

\section{MODELING THE MULTI-EPOCH MODULATION}

The above model was coded in FORTRAN and fully implemented as an additive model with 10 parameters in the XSPEC spectral fitting software (Arnaud 1996). Specifically, 
TABLE 1

Spectral Results as a Function of the NS Radius - Model Fits for $\mathbf{N}_{\mathrm{H}}=6.8 \times 10^{21} \mathrm{~cm}^{-2}, \mathbf{D}=3.3 \mathrm{kpc}, \psi=\xi=\gamma=0^{\circ}$ (see text for definitions)

\begin{tabular}{lccccccc}
\hline \hline Parameter & \multicolumn{2}{c}{2003} & \multicolumn{2}{c}{2004} & \multicolumn{2}{c}{2005} & 2006 \\
& Sep 8 & Oct 12 & Mar 11 & Sep 18 & Mar 18 & Sep 20 & Mar 12 \\
\hline
\end{tabular}

\begin{tabular}{|c|c|c|c|c|c|c|c|}
\hline \multicolumn{8}{|c|}{$R=9 \mathrm{~km} ; \chi_{\nu}^{2}(\mathrm{dof})=1.09(1914)$} \\
\hline$k T_{h}(\mathrm{keV})$ & 0.91 & 0.95 & 0.93 & 0.91 & 0.84 & 0.71 & 0.60 \\
\hline$k T_{w}(\mathrm{keV})$ & 0.32 & 0.38 & 0.35 & 0.35 & 0.31 & 0.26 & 0.23 \\
\hline$\beta_{h}(\mathrm{deg})$ & 10.06 & 8.47 & 6.69 & 5.33 & 3.81 & 3.52 & 4.08 \\
\hline$\beta_{w}(\operatorname{deg})$ & 56.3 & 38.3 & 39.3 & 42.3 & 42.3 & 55.1 & 82.1 \\
\hline \multicolumn{8}{|c|}{$R=10 \mathrm{~km} ; \chi_{\nu}^{2}(\mathrm{dof})=1.11(1914)$} \\
\hline$k T_{h}(\mathrm{keV})$ & 0.91 & 0.95 & 0.890 & 0.91 & 0.76 & 0.67 & 0.59 \\
\hline$k T_{w}(\mathrm{keV})$ & 0.36 & 0.40 & 0.33 & 0.35 & 0.27 & 0.24 & 0.22 \\
\hline$\beta_{h}(\mathrm{deg})$ & 8.21 & 7.21 & 5.83 & 4.46 & 4.03 & 3.12 & 3.11 \\
\hline$\beta_{w}(\mathrm{deg})$ & 35.8 & 29.4 & 34.6 & 30.2 & 43.8 & 51.9 & 66.5 \\
\hline \multicolumn{8}{|c|}{$R=11 \mathrm{~km} ; \chi_{\nu}^{2}(\mathrm{dof})=1.15(1914)$} \\
\hline$k T_{h}(\mathrm{keV})$ & 0.89 & 0.90 & 0.87 & 0.83 & 0.75 & 0.67 & 0.56 \\
\hline$k T_{w}(\mathrm{keV})$ & 0.35 & 0.37 & 0.32 & 0.29 & 0.27 & 0.24 & 0.21 \\
\hline$\beta_{h}(\mathrm{deg})$ & 7.05 & 6.98 & 5.25 & 4.37 & 3.44 & 2.85 & 3.27 \\
\hline$\beta_{w}(\mathrm{deg})$ & 31.3 & 27.8 & 31.5 & 36.3 & 37.1 & 43.8 & 61.3 \\
\hline \multicolumn{8}{|c|}{$R=12 \mathrm{~km} ; \chi_{\nu}^{2}(\mathrm{dof})=1.09(1914)$} \\
\hline$k T_{h}(\mathrm{keV})$ & 0.85 & 0.86 & 0.86 & 0.82 & 0.71 & 0.63 & 0.59 \\
\hline$k T_{w}(\mathrm{keV})$ & 0.32 & 0.34 & 0.32 & 0.29 & 0.25 & 0.24 & 0.22 \\
\hline$\beta_{h}(\operatorname{deg})$ & 7.06 & 6.39 & 5.01 & 4.22 & 3.45 & 2.53 & 2.27 \\
\hline$\beta_{w}(\mathrm{deg})$ & 33.8 & 28.1 & 28.6 & 32.1 & 37.8 & 41.2 & 45.3 \\
\hline
\end{tabular}

\begin{tabular}{lcllllll}
\hline \multicolumn{7}{c}{$R=13 \mathrm{~km} ; \chi_{\nu}^{2}(\mathrm{dof})=1.10(1914)$} \\
\hline$k T_{h}(\mathrm{keV})$ & 0.83 & 0.85 & 0.82 & 0.76 & 0.69 & 0.61 & 0.53 \\
$k T_{w}(\mathrm{keV})$ & 0.31 & 0.33 & 0.29 & 0.26 & 0.24 & 0.23 & 0.20 \\
$\beta_{h}(\mathrm{deg})$ & 6.35 & 5.84 & 4.81 & 4.14 & 3.29 & 2.44 & 2.83 \\
$\beta_{w}(\mathrm{deg})$ & 31.4 & 26.5 & 29.6 & 36.7 & 37.9 & 39.2 & 49.2 \\
\hline \multicolumn{7}{c}{$R=14 \mathrm{~km} ; \chi_{\nu}^{2}(\mathrm{dof})=1.14(1914)$} \\
\hline$k T_{h}(\mathrm{keV})$ & 0.80 & 0.81 & 0.79 & 0.74 & 0.67 & 0.59 & 0.52 \\
$k T_{w}(\mathrm{keV})$ & 0.28 & 0.30 & 0.27 & 0.23 & 0.23 & 0.22 & 0.19 \\
$\beta_{h}(\mathrm{deg})$ & 6.16 & 5.79 & 4.49 & 4.07 & 3.23 & 2.40 & 2.45 \\
$\beta_{w}(\mathrm{deg})$ & 33.26 & 28.9 & 30.7 & 43.5 & 38.5 & 38.1 & 47.9 \\
\end{tabular}

NOTE. - Uncertainties on each spectral parameter are consistent with those reported for the fits in Table 3.

these parameters are the temperatures $\left(k T_{h}, k T_{w} ; \mathrm{keV}\right)$ and subtended angular sizes $\left(\beta_{h}, \beta_{w}\right.$; degs) of the hot spot and warm ring, respectively, the viewing $(\psi)$ and hot spot $(\xi)$ angles w.r.t the spin-axis (in degrees), the rotation phase $(\gamma$; cycles), and finally, the NS radius $(R ; \mathrm{km})$ and distance $(D$; $\mathrm{kpc})$. The XSPEC normalization is set to unity so that the flux is fixed by the distance and stellar radius, which implicitly takes into account all relativistic effects previously noted in $\S 2$. In the following spectral fits the pulsar distance is set to $D=3.3 \mathrm{kpc}$, based on radio pulse dispersion (Camilo et al. 2006), and consistent with the measurement derived from HI absorption (Minter et al. 2007).

This model allows us to predict the energy dependent modulation, and use it to determine the viewing geometry and beaming pattern of the emitted radiation that best match the observations at different epochs. We assume both to be temporal invariant, i.e. no noticeable precession changes with time. In principle, the neutron star radius, because of general relativistic effects, could be uniquely determined; however, in practice the presence of noise does not allow for this most interesting of constraints. In the following, we apply this model to the data sets presented in Gotthelf \& Halpern (2007), which fully described their preparation. We first present the spectral fits $(\$ 3.1)$ using the full model, assuming a face-on geometry $(\alpha=0)$, and then constrain the overall emission geometry of the system ( $\$ 3.2)$ by modeling the observed pulse modulation in 6 energy bands over time.

All spectral fitting are done in the $0.7-10 \mathrm{keV}$ spectral band assuming no beaming initially, as this is not an important effect spectrally. However, some degree of anisotropy of the radiation is found to be necessary in our model to reproduce the observed modulation (\$3.2).

\subsection{Spectral analysis}

We started by fitting the phase-averaged XMM-Newton spectra for the 7 epochs simultaneously using our model for the pulsar emission geometry. Since the viewing geometry is not known a priori, we assume the simplest choice, that we are looking directly down the co-aligned rotation axis and magnetic pole ( $\alpha=0$, see Eq. 1$)$. This has the practical benefit of allowing the model code to run substantially faster since, for this special case of $\alpha=0$, the integration is simpler and only one call to the routine is needed for the computation of the phase-averaged spectrum. Across all epochs, all parameters are linked with the exception of the set of 4 epoch variable parameters $\left(k T_{w}, k T_{h}, \beta_{w}, \beta_{h}\right)$. Initial fits were used to determine the nominal column density of $N_{\mathrm{H}}=6.8 \times 10^{21} \mathrm{~cm}^{-2}$, which was subsequently fixed to this value.

An important technical issue for these fits is the degree of degeneracy between the radius $R$ and the four epoch variable parameters $\left(k T_{w}, k T_{h}, \beta_{w}, \beta_{h}\right)$. These 5 parameters overdetermine the fit, unlike fits using a double blackbody model. Without fixing the radius there is no unique solution, and thus we consider a range of possible values between $9 \leq R \leq$ $14 \mathrm{~km}$, in $1 \mathrm{~km}$ increments. These results are presented in Table 1 and show a similar trend to those reported by Gotthelf \& Halpern (2007) using the double-blackbody model. In both cases, the hot components is found to steadily decrease in size over time, while the warm component increases (with the exception of the first data point).

In our model, the radius of the star is not just a simple normalization. This is due to the introduction of gravitational redshift effects. Unlike for the non-relativistic case, the inferred temperatures of the spots increase as the radius of the star becomes smaller. Two counteracting effects, both due to flux conservation influence the spot size - gravitational redshift tends to decrease the inferred emission area in the more relativistic (smaller) stars (due to the higher inferred temperatures); on the other side, for a fixed distance between the star and the observer, the spot angular size increases on smaller stars. For the values of the fit parameters here, the latter effect tends to dominate over the former. Over the sampled range, we do not find evidence for a preferred radius, based on the $\chi^{2}$ measurements.

As discussed above, the results of our spectral fits using the above model show a similar trend to those reported by Gotthelf \& Halpern (2007) using a double-blackbody model. The hot components steadily decrease in size over time, while the warm component increases (except for the first data point). We are aware of the importance of a possible third emission component from the rest of the NS surface, perhaps the quiescent emission, initially masked by the significant extra flux from the warm component activated by the outburst. However, we are unable to resolve any additional component, which is not required by the spectral fits. If the last few data 
TABLE 2

Minimum $\chi_{\nu}^{2}$ as a Function of Beam Indices $\left(\mathbf{n}_{\mathbf{w}}, \mathbf{n}_{\mathbf{h}}\right)$ and NS Radius

\begin{tabular}{ccccccc}
\hline \hline$n_{w}, n_{h}$ & & \multicolumn{5}{c}{ Minimum $\chi_{\nu}^{2}$} \\
& $R=9 \mathrm{~km}$ & $R=10 \mathrm{~km}$ & $R=11 \mathrm{~km}$ & $R=12 \mathrm{~km}$ & $R=13 \mathrm{~km}$ & $R=14 \mathrm{~km}$ \\
\hline 0,0 & 4.06 & 4.91 & 3.97 & 4.62 & 3.85 & 2.86 \\
0,1 & 0.86 & 1.07 & 1.13 & 0.96 & 0.94 & 0.99 \\
0,2 & 3.17 & 3.25 & 3.74 & 3.02 & 3.29 & 2.83 \\
1,1 & 2.51 & 3.50 & 2.55 & 2.64 & 1.81 & 1.17 \\
\hline
\end{tabular}

NOTE. - Minimum reduced $\chi_{\nu}^{2}$ after comparing model and observed PFs over the $\xi, \psi$-space, with the NS radius and beaming indices held fixed at the given values. Only the first 4 epochs were included in this analysis.

sets are substantially affected by this potential third component, our pulse profile modeling of those data could be incomplete. Therefore, for the second part of our analysis, we rely on the first 4 data sets alone, during which the emission from the two components dominates over that from the NS surface (whose quiescent level was measured with ROSAT prior to the outburst).

\subsection{Modulation analysis}

Starting with the best spectral fit model parameters presented in Table 1 , obtained for $\xi=\psi=0$, we now searched for the best values of $\xi$ and $\psi$ needed to reproduce the observed magnitude of the pulse modulation across the first 4 epochs, as measured by Gotthelf \& Halpern (2007). Given the uncertainties in the data, we limit our modeling to that of the pulse modulation, rather than the full pulse profile. The pulsed fractions were determined in six energy bands ${ }^{5}$ at each epoch. For each value of the NS radius $(R)$ fitted for in $\$ 3.1$, we computed the modulation (defined below) over the grid of angles $\xi, \phi \leq 90 \mathrm{deg}$, in 1 degree intervals. For each value on the grid, the model predictions were compared with the data. Notice that the flux depends on the angles $\xi$ and $\psi$ only through the parameter $\alpha$ in Eq.(1), and therefore it is symmetric with respect to an exchange of $\xi$ and $\psi$. The magnitude of the model modulation is defined as

$$
P F=\frac{F_{\max }-F_{\min }}{F_{\max }+F_{\min }} .
$$

In the geometry that we are considering, the maximum and minimum fluxes, $F_{\max }$ and $F_{\min }$, correspond to phases $\gamma=0$ and $\gamma=\pi$, respectively. Both fluxes are integrated over the given energy bands.

The results of these fits show that it is not possible to reproduce the observed modulation if the emission pattern of both the hot and warm component is isotropic. The introduction of General Relativity effectively suppresses the modulation to below that observed, for any reasonable assumed NS radius. We ascribe the observed modulation to additional anisotropic emission from the thermal regions and test this assumption using a simple model of cosine beaming described in $\$ 2$. For each value of the radius, we ran through the $(\psi, \xi)$ grid of models for different combinations of the beaming parameters $n_{w}$ and $n_{h}$ of the warm and hot components, respectively. More specifically, we varied $n_{w}, n_{h}$ between 0 and 2 , in increments of 0.5 (note that the softer component is less modulated than the harder one). The fact that the warm component dominates in flux in the softest energy band, while the hot component dominates in the hardest energy band, allows us to constrain

\footnotetext{
5 \{0.5-1; $1-1.5 ; 1.5-2 ; 2-3 ; 3-5 ; 5-8\} \mathrm{keV}$.
}

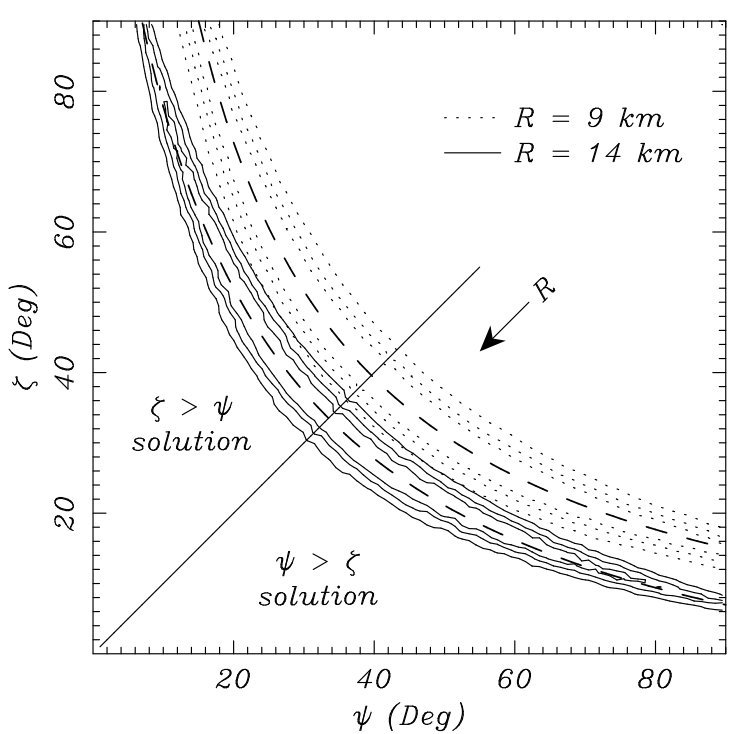

FIG. 2.- Reduced chi-square $\left(\chi_{\nu}^{2}\right)$ maps obtained by comparing modulation data and model described in the text for a range of viewing angles $\psi$ and $\xi$. The $68 \%, 90 \%$ and $99 \%$ confidence levels are shown for the best match to the observed pulsed fractions using the beaming patterns $n_{w}=0, n_{h}=1$, for $R=9 \mathrm{~km}$ and $R=14 \mathrm{~km}$. The results are clearly degenerate with respect to an interchange of $(\xi, \psi)$. For the adopted model, the locus of minimum $\chi_{\nu}^{2}$ depends only on radius and lies along a line $(\xi+\beta(R))(\psi+\beta(R)) \approx$ constant (dashed lines). The minimum $\chi_{\nu}^{2}$ for each value of the radius is reported in Table 2 , and the angles $(\psi, \xi)$ to which this minimum corresponds vary slightly with radius.

the degree of anisotropy of these two components independently. For each set of angles $\psi$ and $\xi$ on the grid, we computed the reduced $\chi_{\nu}^{2}$ and kept track of its minimum. Table 2 reports the minimum $\chi_{\nu}^{2}$ that was obtained for a few representative values of the beaming parameters.

With the introduction of beaming, we are able to identify a set of model parameters that is able to reproduce the observed modulation in the first 4 epochs. The main results from our modeling can be summarized as follows:

i) The modulation of the hot spectral component requires an anisotropic radiation pattern. For a $\cos ^{n_{h}} \delta$ emission profile, the best match to the data is obtained with $n_{h} \approx 1$.

ii) No similar beaming is required to model the warm component modulation (i.e. $n_{w} \sim 0$ ).

iii) We constrain the emission geometry by identifying allowed and forbidden regions in the $\psi-\xi$ parameter space.

iv) No NS radius is strongly preferred by the data. However, the range of most preferred values of $\psi$ and $\xi$ varies with the radius of the star.

For the optimal beaming parameters, $n_{w}=0$ and $n_{h}=1$, we plot the $\chi_{\nu}^{2}$ map computed for the analysis of Table 2 . Figure 2 displays the $68 \%, 90 \%$ and $99 \%$ confidence levels drawn for the two extreme values of radii considered $(R=9,14 \mathrm{~km})$. As described above, this map is produced by comparing the model and observed modulation over a range of possible $(\xi, \psi)$ angle pairs, for our best fit spectral model parameters. The range of allowed solutions defines a locus of points in the $\xi-\psi$-plane along the line $(\xi+\beta(R))(\psi+\beta(R))=c$, were $c$ is, to zeroth order a constant for the given model (it 
varies by $\lesssim 9 \%$ between the two extreme values of radii considered here), while $\beta(R)$ contains the strong dependence on the NS radius. A linear regression fit yields the relations $\beta=1.64 R_{\mathrm{km}}-6.16$ and $c=40.65 R_{\mathrm{m}}+1935$, where $R_{\mathrm{km}}$ is the radius of the star in $\mathrm{km}$. The elongated shape of the contour plots shows that the two angles $\psi$ and $\xi$ are highly correlated in the fit. This is a result of the fact that the PF depends on a combination of these two angles.

The dependence on the NS radius is clearly seen in Figure 2 For each given value of $\psi$, the best fit loci moves toward larger values of $\xi$ as the radius of the star gets smaller. The contour levels for intervening values of the radii fall in-between those shown. This trend with radius can be understood as follows. As the radius decreases, the larger angular sizes and temperatures conspire to decrease the PF for the same viewing angles $\psi$ and $\xi$. In addition, the gravitational effect of light bending reduces the modulation even further for small stars. In order to reproduce the same observed $\mathrm{PF}$ for a given $\psi$, a correspondingly larger $\xi$ is therefore needed for smaller stars since, for any given value of each of these two angles, a larger value of the other produces a larger modulation. This is the reason for the shift of the confidence levels toward larger values of $\xi$ for a given $\psi$, when the radius of the star gets smaller.

Figure 3 compares the data and model modulation for the $(\psi, \xi)$ values that yield minimum $\chi_{\nu}^{2}$ for the case $R=12 \mathrm{~km}$, $n_{w}=0, n_{h}=1$. We show the results for two cases, one using all seven epoch data sets, and one for the case of the first four data sets only. In the former case, the minimum corresponds to $\psi=49^{\circ}$ and $\xi=24^{\circ}$, while in the latter, it occurs for $\psi=53^{\circ}$ and $\xi=23^{\circ}$. We find excellent agreement between data and model modulation for the first four observations alone; however, the later data sets show increasing discrepancies, noticeably increasing the overall contribution to $\chi^{2}$. This confirms the suspicion that, at later times, the emitted radiation acquires an unpulsed (or very mildly pulsed) contribution from the surface of the star. Our spectral fits, as described above, do not resolve this underlying stellar component, and therefore the spectral parameters close to quiescence might not be as representative of the underlying physical parameters of the system. However, the constraints on the viewing and emission geometry that we derive using the first 4 data sets alone (cfr. Table 2 and Fig.2) can be considered robust, since the early data sets are basically unaffected by the presence of the star underlying emission.

As a final step in our analysis we consider the validity of our initial method of assuming a face-on spectrum to derive the spectral model parameters that are then used to compute the modulation. As discussed in $\S 3.1$, the original fits were generated assuming $\psi=\xi=0$ for simplicity, prior to determining the observational geometry of the pulsar system. We now show that this is an excellent assumption by refitting to the data the spectrum assuming the specific case of $\psi=53^{\circ}$ and $\xi=23^{\circ}$, and then recomputing the modulations. The best fit spectral parameters are reported in Table 3 and shown in Figure 4. For these spectral parameters the model modulation is shown in Figure 3 as the dashed-line for the case of the first four data sets. The results are identical within the statistical uncertainty in the data.

Similarly, we performed a test in order to assess the validity of our method of analysis which separated the spectral and timing studies and used, as spectral parameters for the timing analysis, those obtained from a phase-averaged fit to the spectrum. We again considered the specific case of $\psi=53^{\circ}$ and $\xi=23^{\circ}$, and extracted phase-resolved spectra by dividing the observed spectrum into 5 equally spaced bins. We then fitted the model to the two bins centered on the maximum and minimum of the flux, respectively. We found that the temperature at flux minimum is lower than the temperature at flux maximum by $\lesssim 10 \%$ for all XMM epochs with the exception of the first one, where the difference is $\sim 20 \%$. However, this difference is within one $\sigma$ of the combined uncertainty in temperature for all epochs. Therefore, our adopted method is quite robust within the statistical uncertainty of the data.

\section{DISCUSSION}

The time-dependent spectrum and pulse modulation of the transient magnetar XTE J1810-197 provide a unique diagnostics of its emission properties and geometry. Under the assumption that the post-burst emission is described by two thermal components, as early analysis of this object suggested, we have been able to extract information on some of the physical properties of the star, through a detailed modeling of the combined spectra and pulsed modulation together.

We found that, while the phase-averaged spectral fits alone are degenerate with respect to the emission pattern of the radiation, including modeling of the energy-dependent pulsed flux allows us to constrain the properties of the emission region. In particular, since the warm component dominates in the lowest energy band, while the hot component dominates in the highest energy band, the PFs are able to determine the degree of anisotropy of these two components independently. We found that the warm component is best described by an isotropic emission pattern, while the hot component is well represented by an emission pattern of pencil type, $f(\delta) \propto \cos \delta$, where $\delta$ is the angle that the emitted photons make with respect to the normal to the surface of the star. The different type of radiation pattern required by the low and the high energy components could be seen as an indirect confirmation of our assumption that the contribution from these two energy bands does indeed come from different components. Beaming of radiation in the direction of the magnetic field is predicted by models of magnetized atmospheres in the limit of high magnetic fields (e.g. van Adelsberg \& Lai 2006). Since the hot spot is produced in a region much smaller than that of the warm component, it is more likely to find a configuration with parallel field lines in the hot region (and most likely perpendicular to the surface, which favors the heat flow), than in the warm region. The latter might rather encompass regions with different orientations of the magnetic field, hence resulting in an overall more homogeneous radiation pattern.

The strongest constraints that we derived from our analysis are on the geometry of the star. In particular, we determined the allowed regions for the angle $(\xi)$ between the spot axis and the rotation axis, and the angle $(\psi)$ between our line of sight and the rotation axis. These two angles determine the minimum and maximum angles between the line of sight and the spot axis, given respectively by $\alpha_{\min }=\xi-\psi$ and $\alpha_{\max }=$ $\xi+\psi$. We find that, while the range of $\alpha_{\min }$ is compatible with very small angles (including zero), however $\alpha_{\max }$ must always be large, $\gtrsim 60^{\circ}$ within $3 \sigma$ confidence level for any value of the star radius. Being able to rule out to a high confidence level small viewing angles $\alpha$ for the entire rotation period of the star bears important implications for models of the observed radio emission from this object. In fact, Camilo et al. (2007a) showed that the peaks of the radio and the X-ray pulses are aligned, suggesting that the footpoints of the active magnetic field lines on which radio emission is generated are also the locations of the concentrated heating that is responsible for 

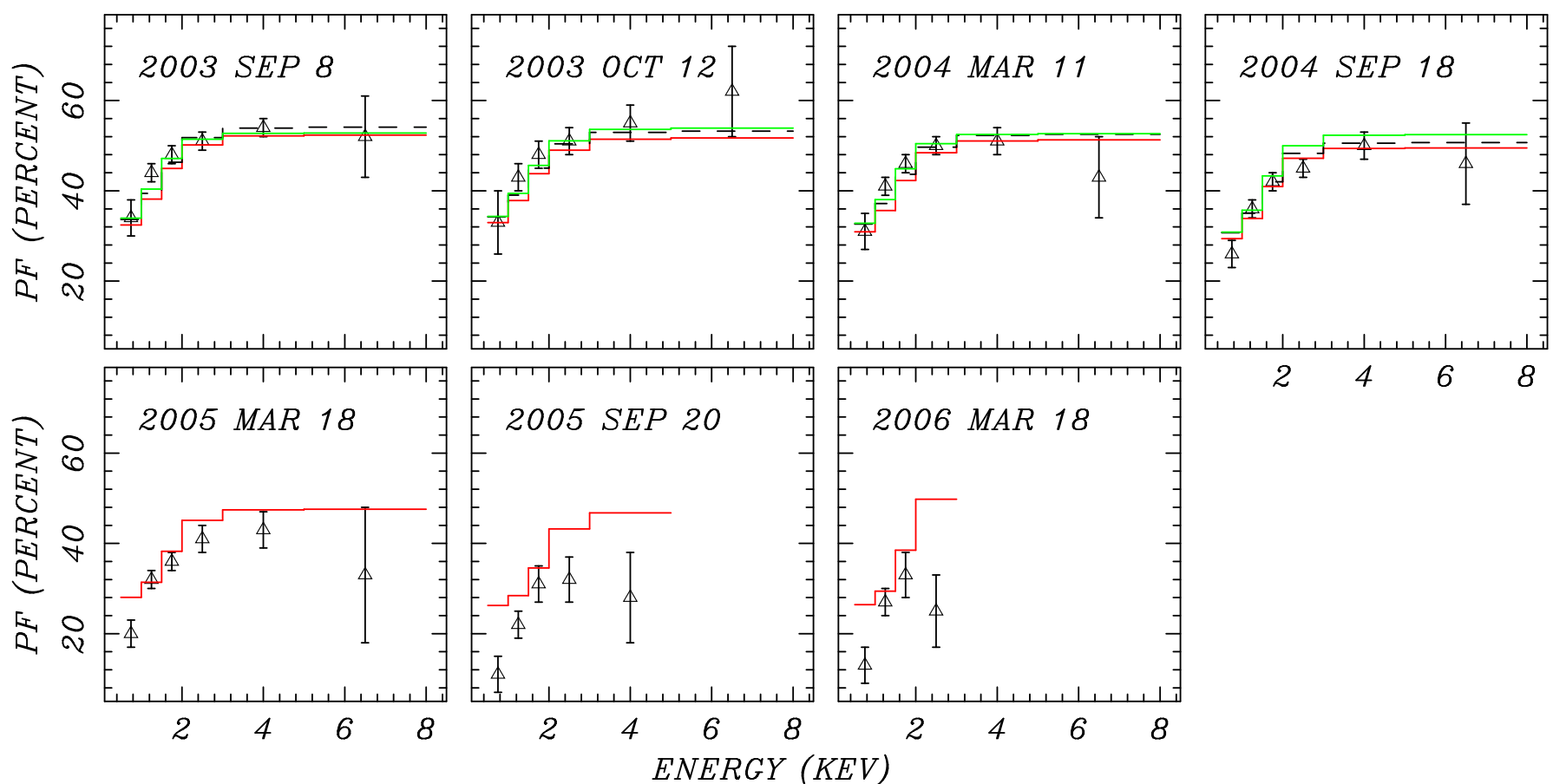

FIG. 3.- Comparison between the measured pulsed fraction (PF) and that predicted by our model for one particular set of best-fit parameters determined for a $R=12 \mathrm{~km}$ neutron star. Results are shown for the best fit model using all seven data sets (red line; $\psi=49^{\circ}$ and $\xi=24^{\circ}$ ) and for the first four data sets only (green line; $\psi=53^{\circ}$ and $\xi=23^{\circ}$ ). At the later times, the model is seen to deviate significantly from the data. A likely explanation is the increased contribution of the unmodeled emission from the stellar surface over time compared to the modeled flux. In the first 4 data sets, the dashed line is to be compared to the green line, showing the effect of using the face-on spectra instead of the iterated spectra for the given viewing geometry (see text for details). Each data point is drawn in the middle of the corresponding energy band, for those bins with sufficient photons.

TABLE 3

Spectral Results for Radius $\mathbf{R}=\mathbf{1 2} \mathbf{~ k m}$ and Viewing Angles $\psi=\mathbf{5 3} \mathrm{deg}$, and $\xi=\mathbf{2 3} \mathrm{deg}$;

$\mathrm{D}=3.3 \mathrm{kpc}, \mathrm{N}_{\mathrm{H}}=6.8 \times 10^{21} \mathrm{~cm}^{-2}$

\begin{tabular}{lccccccc}
\hline \hline Parameter & \multicolumn{2}{c}{2003} & \multicolumn{2}{c}{2004} & \multicolumn{2}{c}{2005} & 2006 \\
& Sep 8 & Oct 12 & Mar 11 & Sep 18 & Mar 18 & Sep 20 & Mar 12 \\
\hline$k T_{h}(\mathrm{keV})$ & $0.84_{-0.02}^{+0.03}$ & $0.87_{-0.04}^{+0.04}$ & $0.85_{-0.03}^{+0.01}$ & $0.82_{-0.02}^{+0.02}$ & $0.73_{-0.02}^{+0.03}$ & $0.63_{-0.04}^{+0.03}$ & $0.53_{-0.03}^{+0.04}$ \\
$k T_{w}(\mathrm{keV})$ & $0.30_{-0.03}^{+0.68}$ & $0.34_{-0.06}^{+0.11}$ & $0.31_{-0.03}^{+0.01}$ & $0.29_{-0.04}^{+0.04}$ & $0.26_{-0.02}^{+0.06}$ & $0.23_{-0.03}^{+0.01}$ & $0.21_{-0.01}^{+0.02}$ \\
$\beta_{h}(\mathrm{deg})$ & $8.0_{-0.2}^{+0.2}$ & $7.1_{-0.2}^{+0.7}$ & $5.5_{-0.1}^{+0.3}$ & $4.52_{-0.08}^{+0.06}$ & $3.8_{-0.2}^{+0.4}$ & $3.02_{-0.05}^{+0.04}$ & $3.4_{-0.8}^{+0.5}$ \\
$\beta_{w}(\mathrm{deg})$ & $39_{-2}^{+2}$ & $30_{-3}^{+2}$ & $31.0_{-1.7}^{+0.7}$ & $33.9_{-1.0}^{+0.5}$ & $37.7_{-0.1}^{+0.7}$ & $44_{-3}^{+10}$ & $60_{-3}^{++.5}$
\end{tabular}

NoTE. - Uncertainties in spectral parameters are 90\% confidence for two interesting parameters. The $\chi_{\nu}^{2}$ of the fit is 1.08 for 1692 dof.

the enhanced X-ray emission, This means that, even if the radio emission is likely produced at much higher altitudes on the surface of the star than the X-ray emission, however the axis where the two emissions peak is the same (or very close).

Attempts to constrain the viewing angles of XTE J1810-197 using radio polarimetry were made by Camilo et al. (2007b). They found two configurations likely, one with $\xi \sim 70^{\circ}$ and $\alpha_{\min } \sim 20^{\circ}-25^{\circ}$, and another with $\xi \sim 4^{\circ}$ and $\alpha_{\min } \sim 4^{\circ}$. Our fits rule out the second configuration to a high significance level. The value of $\alpha_{\min } \sim 20^{\circ}-25^{\circ}$ on the other hand is perfectly compatible with our results, albeit it requires $\xi \sim 60^{\circ}$ if $R=9 \mathrm{~km}$ and $\xi \sim 50^{\circ}$ if $R=14 \mathrm{~km}$. Although our confidence levels are close to one of the two solutions of Camilo et al. (2007b), we cannot make a formal statistical comparison with their results, since they do not have a reliable estimate of the parameter uncertainties from their radio measurements (F.
Camilo, priv. comm.).

As discussed by Camilo et al. (2007b), the observed wide radio pulse profile of $\approx 0.15 P$ can be explained by either a model in which the magnetic and rotation axes are almost aligned, or by a model in which the emission height is very large. Our results strongly rule out the first scenario, hence implying a large emission height. This, in turn, implies a large opening angle of the beam (Gil et al. 1984), comparable to that observed in young pulsars (Johnston \& Weisberg 2006). These characteristics of the radio emission, if common in magnetars, make more stringent the limits on the radio for the greatest majority of the objects that have not been detected in this waveband, and leave even more open the question of what is that makes some magnetars different.

We thank Jules Halpern, Andrei Beloborodov and Fernando Camilo for stimulating discussions on several aspects of this 


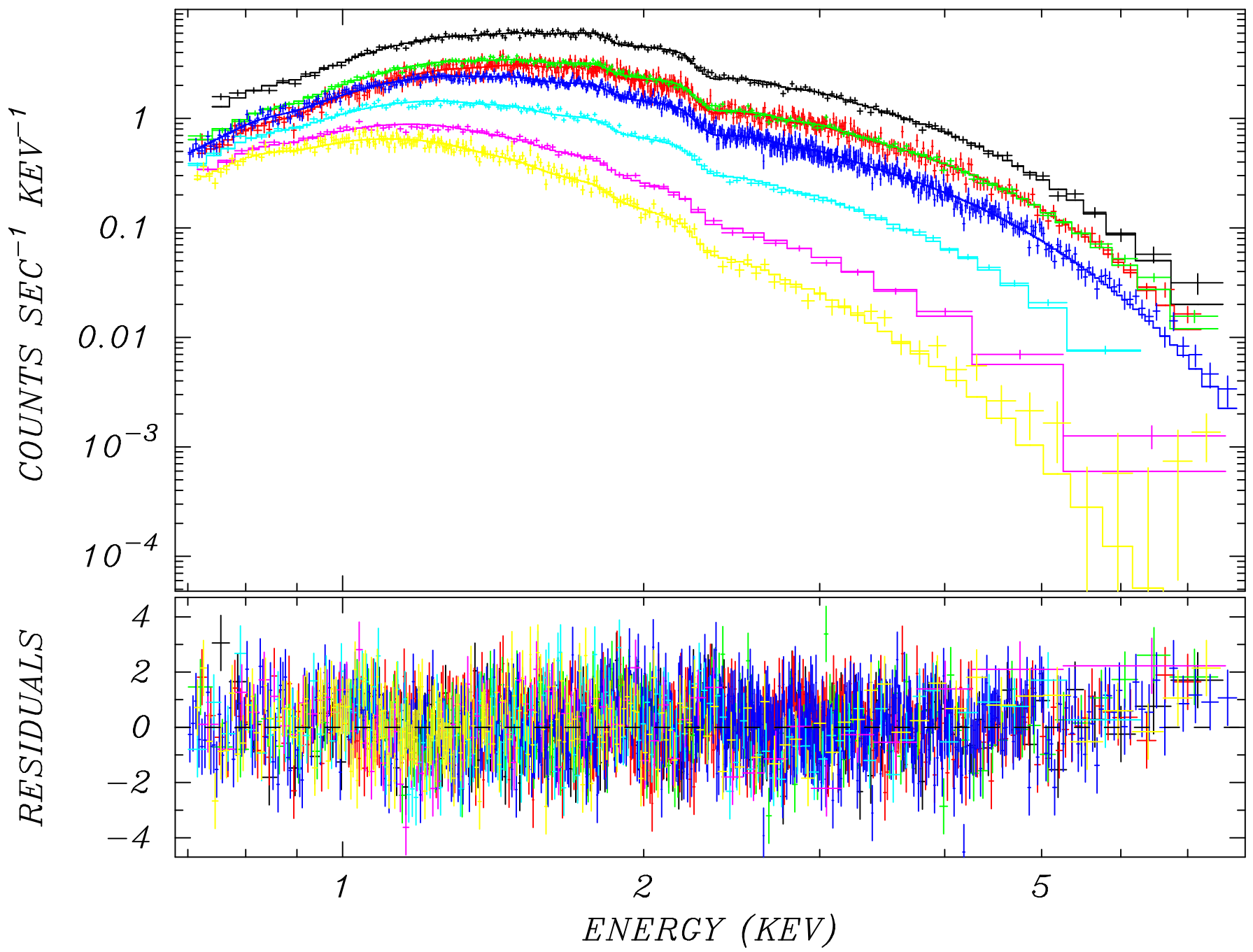

FIG. 4.-XMM-Newton phase-averaged spectra of XTE J1810-197 obtained at 7 epochs fitted with the model presented in the text, for the specific case of $R=12 \mathrm{~km}, \psi=53^{\circ}$ and $\xi=23^{\circ}$. The parameters for the best fit model are reported in Table 3. The lower panel shows the collected residuals to this fit for each spectrum.

work. RP thanks Columbia University for the kind hospitality during the several visits made while this work was carried out.
We also thank the referee for his/her insightful and helpful comments on our manuscript.

\section{REFERENCES}

Arnaud, K.A., 1996, Astronomical Data Analysis Software and Systems V, eds. Jacoby G. and Barnes J., p17, ASP Conf. Series volume 101

Baring, M. G. \& Harding, A. K. 1998, ApJ, 507L, 55

Beloborodov, A. 2002, ApJ, 566L, 85

Beloborodov, A. M. \& Thompson, C. 2007, ApS\&S, 308, 631

DeDeo, S., Psaltis, D., Narayan, R. 2001, ApJ, 559, 346

Duncan, R. C. \& Thompson, C. 1992, ApJ, 392, L9

Camilo, Fernando, Ransom, S. M., Halpern, J. P., Reynolds, J., Helfand, D. J., Zimmerman, N., Sarkissian, J. 2006, Nat., 442, 892

Camilo, F. et al. 2007a, ApJ, 663, 497

Camilo, F., Reynolds, J., Johnston, S., Halpern, J. P., Ransom, S. M., van Straten, W. 2007b, ApJL, 659, 37

Gil, J. A. Gronkowski, P. \& Rudnicki, W. 1984, A\&A, 132, 312

Gotthelf, E. V., Halpern, J. P., Buxton, M., Bailyn, C. 2004, ApJ, 605, 368

Gotthelf, E. V., Halpern, J. P. 2005, ApJ, 632, 1075

Gotthelf, E. V., Halpern, J. P. 2007, Ap\&SS, 308, 79

Gotthelf, E. V., Halpern, J. P., Buxton, M. \& Bailyn, C. 2004, ApJ, 605, 368

Guver, T., Ozel, F., Gogus, E., \& Kouveliotou C. 2007, ApJL, 676, 73

Halpern, J. P.; Gotthelf, E. V. 2005, ApJ, 618, 874
Halpern, J. P., Gotthelf, E. V., Becker, R. H., Helfand, D. J., White, R. L. 2005, ApJ, 632L, 29

Ibrahim, A. I. et al. 2004, ApJ, 609L, 21

Israel, G. et al. 2004, ApJ, 603L, 97

Johnston, S., Weisberg, Joel M. 2006, MNRAS, 368,1856

Kaspi, V. M. 2007, Ap\&SS, 308, 1

Lyutikov, M. \& Gavriil, F. P. 2006, MNRAS, 368, 690

Minter et al. 2007, ApJ, submitted

Page, D. 1995, ApJ, 442, 273

Pechenick, K. R., Ftaclas, C., Cohen, J. M. 1983, ApJ, 274, 846

Perna, R., Heyl, J. S., Hernquist, L. E., Juett, A. M., Chakrabarty, D. 2001a, ApJ, 557, 18

Perna, R., Heyl, J., Hernquist, L. 2001b, ApJ, 553, 809

Rea, N., Zane, S., Lyutikov, M., Turolla, R. 2007, Ap\&SS, 308, 61

Skinner, S.L., Perna, R. \& Zhekov, S. A. 2006, ApJ, 653, 587

Thompson, C. \& Duncan, R. C. 1996, ApJ, 473, 322

van Adelsberg, M., Lai, D. 2006, MNRAS, 373, 1495 